\title{
Gran Santa Fe (Argentina): metrópoli de segundo orden. Problemáticas y dinámicas recurrentes
}

\author{
Mirta Soijet \\ Jaquelina Paola Santiago \\ Victoria Ivón García
}

Universidad Nacional del Litoral, Argentina

Recibido: 24 de mayo del 2017 / Aprobado: 10 de septiembre del 2017

doi: 10.26439/limaq2018.n004.2257

La Región Metropolitana Santa Fe-Paraná pertenece a una particular categoría que comienza a ser reconocida en el análisis territorial como metrópoli de segundo orden. Una serie de procesos recientes, resultado de instancias de reestructuración productiva y social, permiten orientar este trabajo al desarrollo de aspectos y problemáticas derivados de los requerimientos que plantean los vínculos fisicos y funcionales de las diferentes jurisdicciones. Se indagan sus causas, se presenta la situación actual y se apunta a definir las metodologías y criterios de actuación para constituir un modelo de organización del territorio con equilibrio ambiental, donde se evite la segregación y fragmentación, se preserven las actividades agrícolas de borde y se protejan los paisajes.

metrópolis de segundo orden, problemáticas urbanas, extensión metropolitana

The Great Santa Fe (Argentina): a second-order metropolis.

\section{Recurring problems and dynamics}

The Santa Fe-Paraná Metropolitan Region is part of a particular category that begins to be recognized in territorial analysis as a second-order metropolis. A series of recent processes, resulting from instances of productive and social restructuring, allow this work to be aimed at developing aspects and problems derived from the needs posed by the physical and functional links of different jurisdictions. We investigate its causes, present the current situation, and define the methodologies and action criteria to formulate an environmentally-balanced territorial organization model, preventing segregation and fragmentation, preserving border agricultural activities, and protecting landscapes.

second-order metropolis, urban problems, metropolitan expansion 


\section{INTRODUCCIÓN: PRESENTACIÓN DEL SISTEMA REGIONAL}

La Región Metropolitana Santa Fe-Paraná (RMSF-P) se presenta como un ámbito urbano-rural que en el 2010 alcanzó (y superó) el millón de habitantes. Esta situación motivó nuevas indagaciones en función de la capacidad para iniciar la consolidación de este espacio metropolitano ${ }^{1}$.

Cabe señalar que la RMSF-P tiene como particularidad la duplicación de su ciudad central y define una configuración bicéfala, conformada por Santa Fe y Paraná y sus hinterlands. Se trata de dos centros urbanos de similar jerarquía e importancia simbólica, circunstancias históricas comunes, cierta complementariedad funcional y un interés de larga data por alcanzar una interacción más intensa y efectiva. Estas localidades están separadas por el río Paraná y conectadas por un túnel subfluvial, inaugurado en 1969; asimismo, se mantiene la perspectiva de una nueva conexión vial, que podría materializarse en la próxima década ${ }^{2}$. Cada núcleo urbano reúne localidades aglomeradas que conforman el Gran Santa Fe y el Gran Paraná. Así, se determina un primer nivel de metropolización, que puede entenderse como el Área Metropolitana Santa Fe-Parana (AMSF-P); y un segundo nivel, la RMSF-P, que completa el sistema con un anillo de ciudades perimetrales con atributos de subcentros y un conjunto de pequeñas localidades en el área interior a tal límite virtual.

1 En ese sentido, el equipo de investigadores, nucleado en el Observatorio Urbanístico del AMSF-P, viene desarrollando actividades desde distintos proyectos de investigación con subsidios de la UNL, la SPU y la ANPCyT, de extensión universitaria y junto con gobiernos locales e instituciones de la sociedad.

2 En el 2014 se realizó el Ilamado a licitación para la elección de la traza vial. La empresa seleccionada fue la única que postuló (ATEC SA) y conformó una UTE. Además, se contrataron los servicios de tres universidades para el monitoreo: la Nacional de Rosario, la del Litoral y la Tecnológica de Paraná. La primera etapa de estudio y selección de la traza ya fue desarrollada, y en estos momentos debería estar concluyendo el proyecto ejecutivo. Sin embargo, la elección de la traza del lado santafesino ha motivado una gran polémica, debido a que no confluyen los intereses del Gobierno municipal con las mejores condiciones de localización de esta. Tal situación ha retrasado los tiempos planeados para las diferentes tareas, y hasta el día de hoy aún hay dudas sobre cuál será la traza definitiva. 


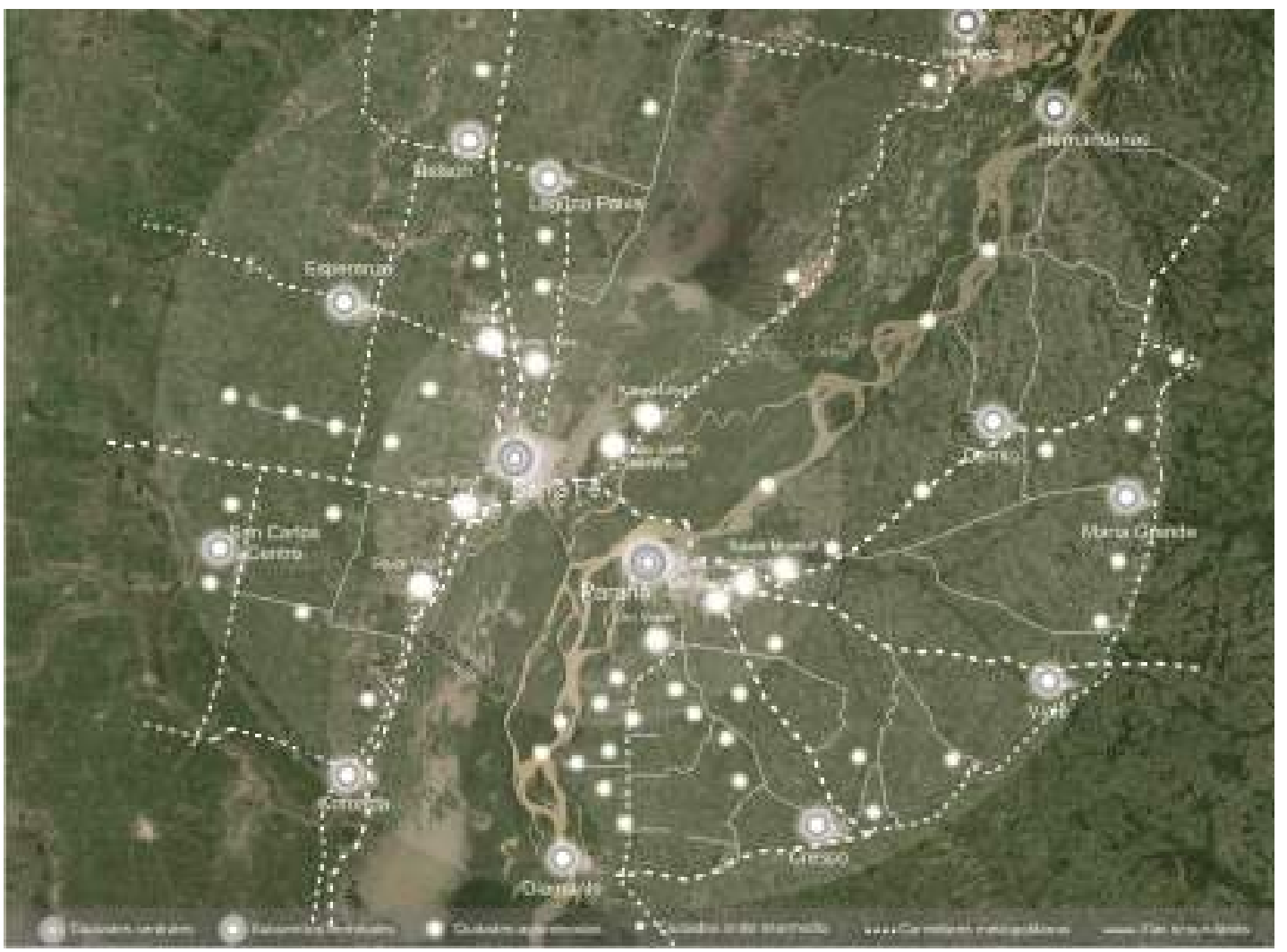

Figura 1

Imagen satelital que muestra el sistema RMSF-P

Fuente: Google Earth

Se trata de un sistema urbano-territorial que, por una parte, está ligado a los espacios rurales y naturales con los que se interrelaciona directamente y, por otra, ejerce funciones de mediación con las redes/ciudades que están fuertemente condicionadas por la globalización económica, cultural y política. Constituye junto con muchos otros casos que vienen siendo estudiados en el 
país ${ }^{3}$ una categoría identificada como metrópoli de segundo orden, metrópoli del interior o metrópoli regional (Ministerio de Planificación Federal, Inversión Pública y Servicios, 2011; Fein, M., 2014; Freitas-Firkowski, O. y Casares, M., 2016). Tal clasificación conlleva, frente a las configuraciones del ámbito nacional (en Argentina: Buenos Aires, Córdoba y Rosario) el reconocimiento de una condición de menor jerarquía, con potencial para la construcción de espacios regionales, con peso propio y que conforma hinterlands de alta interacción y complementación interna.

Las reflexiones siguientes, que pretenden caracterizar esa condición, no se centran en el estadio de un proceso, sino en el reconocimiento del fenómeno. Si bien es cierto que las problemáticas y dinámicas que se describen en esta presentación resultan reconocibles en otras escalas metropolitanas, la diferencia está depositada en las posibilidades y modalidades que son susceptibles de adoptar antes ellas. En otras palabras implica que estas metrópolis de segundo orden tienen potencialidades particulares, vinculadas a su condición de nodos regionales con una magnitud de alrededor de un millón de habitantes que desempeñan funciones económicas relacionadas con el comercio y los servicios a escala provincial/regional, con actividades gubernamentales y servicios; y en las que se reconoce un crecimiento demográfico promedio alto. Por todo ello, muchos de los procesos detallados, como la dispersión urbana, surgieron hace 20 o 30 años, pero su agudización es relativamente reciente. A esto se agrega que tales procesos, en términos cuantitativos, podrían ser relativamente controlables de mediar predisposición e instrumentos para ello.

Después de la presentación de esta particular conformación y de agregar que cada una de las ciudades centrales, Santa Fe y Paraná, pertenecen a distintas provincias (lo que conlleva dificultades comprobadas a la hora de pensar el modo de gestionar el conjunto), este trabajo se centrará en el caso de Santa Fe y su área de influencia (ciudades aglomeradas), asunto que resulta ampliamente demostrativo para los procesos que se intentan presentar y analizar.

3 Como Resistencia y Corrientes (Argentina); Tucumán (Argentina) y Curitiba (Brasil); Viedma y Carmen de Patagones (Argentina); Trelew y Rawson (Argentina). 
En ese sentido, el Gran Santa Fe es relevante en tanto encarna dinámicas que, con mayor o menor intensidad, tienen lugar en otras metrópolis del interior y que se sintetizan en estos procesos: situaciones de colmatación y valorización excesiva del suelo en áreas consolidadas; fragmentación y segregación socioespacial; dispersión de las áreas residenciales hacia la periferia de la ciudad central y en localidades próximas; consumo de suelo productivo; grandes parcelas con usos dominantes de infraestructuras; y equipamientos de escala supraurbana.

\section{GRAN SANTA FE: DINÁMICAS INTERJURISDICCIONALES}

Gran Santa Fe es una aglomeración urbana de aproximadamente 500000 habitantes, con continuidad física e intensos vínculos funcionales. Está formada por Santa Fe como ciudad central; Santo Tomé y Sauce Viejo en el arco suroeste; San José del Rincón y Arroyo Leyes en el arco este; y Monte Vera y Recreo en el área norte.

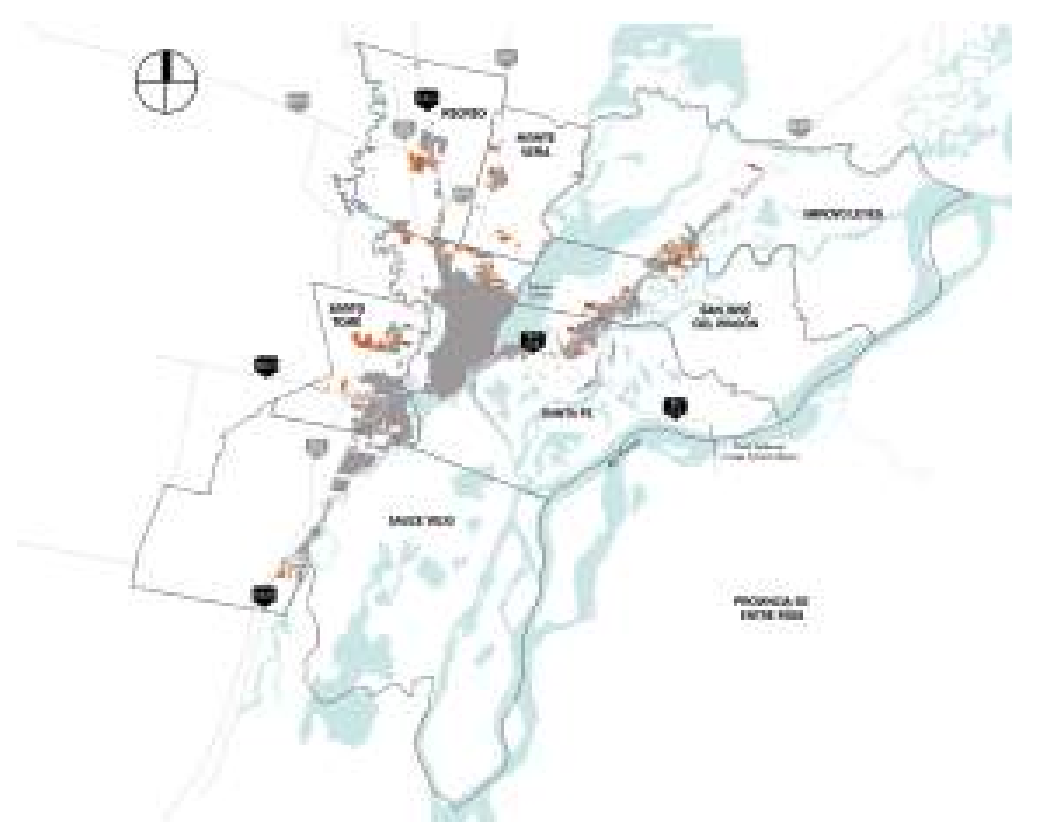

Figura 2

Plano del aglomerado Gran Santa Fe, donde se indican con color las áreas de extensión en la última década (2005-2016)

Elaboración propia 
En lo que respecta a su espacio de sustentación, se trata de un área que tiene un considerable grado de vulnerabilidad, derivado del riesgo hídrico por su condición de planicie en un ancho valle de inundación, afectado por transformaciones antrópicas en su cuenca superior. En lo referente a su dinámica urbanística, el conjunto está inmerso en un proceso de crecimiento que responde a dos modalidades bien diferenciadas y en condiciones tales que alteran fuertemente la sustentabilidad: compactación y extensión.

La modalidad extensión se refiere al caso de las localidades aglomeradas a la ciudad central de Santa Fe (las cuales serán abordadas más adelante con mayor singularidad), que registran un aumento de más del $20 \%{ }^{4}$, tomando un promedio de las diferentes localidades en el tamaño de sus plantas urbanas. Esto responde, en buena medida, a grandes extensiones de nuevos loteos residenciales de baja densidad o usos especializados que la gran ciudad expulsa de sus límites.

En el interior de la ciudad de Santa Fe, por el contrario, se manifiesta fuertemente una tendencia a la compactación de la planta urbana a través de la sustitución edilicia, con fuerte impacto en la densificación de las áreas centrales y pericentrales. Este proceso es conducido principalmente por la iniciativa privada mediante emprendimientos destinados a los sectores medio y medio alto de la sociedad.

Según datos revelados por Martínez (2016, p. 94), si se consideran los edificios construidos desde 1966 - porque con anterioridad a ese año fueron excepcionales-, solo en los últimos 15 años se han registrado el $60 \%$ de estas construcciones.

La tendencia a la verticalización, que se da principalmente en áreas centrales de la ciudad (centro tradicional que se expande hacia los bulevares), se puede constatar también a través de los datos censales del Índice Nacional de Estadísticas y Censos (Indec). Los departamentos lideraron la unidad residencial en tanto se incrementaron en 29,9\% durante los últimos 10 años (Martínez, 2016, p. 94).

4 La superficie de las plantas urbanas del aglomerado (sin incluir Santa Fe) sumaba 4733,4 Ha en el 2005; y se registró una superficie urbana de 5687,2 Ha para el 2016. 
A esto se suma la operación del área portuaria, aledaña al centro y en proceso de reconversión a usos urbanos de servicios y residenciales (supermercado, shopping, hotelería), usos comerciales con requerimiento de grandes superficies, etcétera, lo que ha definido un nuevo sector de atracción ${ }^{5}$.

Tal como se mencionó anteriormente, estas dinámicas pueden ser comprendidas desde una perspectiva más amplia, que tiene que ver con el mercado de tierras; y si bien la compleja relación entre este campo y los de la política económica, la administración y el derecho parten de una cosmovisión que en este articulo no se tiene la intención de indagar, resulta de interés destacar algunas cuestiones específicas en las experiencias, tanto en su condición de investigador como de gestor, de Eduardo Reese (2009). Estas se relacionan directamente con los procesos aquí expuestos, sobre todo por la correspondencia contextual.

El autor sostiene que el ordenamiento territorial conlleva conflictos de intereses, ya que es una actuación pública en un ámbito de propiedad y lógica privadas. A ello se suman la atomización y la disociación del proceso de construcción de la ciudad en dos momentos: el del parcelamiento y el de la efectiva ocupación. Como argumenta Reese (2009), "la lógica del desarrollo urbano argentino fue de crecimiento por adición de suelo, un crecimiento básicamente extensivo con ciertos procesos de segregación socio territorial”.

Por otra parte, resulta verificable la incapacidad estructural del Estado por acompañar o normar ese crecimiento con una producción universal de infraestructuras y equipamientos. Esta deficiencia signó el modelo tradicional y determinó esa particularidad de nuestras ciudades: que presentan áreas centrales fuertemente equipadas, generalmente rodeadas de algún anillo de áreas consolidadas con niveles heterogéneos, pero con presencia de infraestructura y equipamiento; y luego una periferia muy extensa, bastante desarticulada y con frecuencia carente de servicios.

5 En este sentido, resulta significativo su tratamiento desde la zonificación, que —además de establecer el distrito ciudad-puerto- rezonifica sus adyacencias al definir en el borde norte el denominado distrito R11. Este alienta la localización de soluciones habitacionales de alta densidad y actividades compatibles, con el fin de dominar parte del skyline de este frente urbano de relevancia creciente. (Ver Mapa de Modificaciones y Modificaciones Propuestas dentro de la propuesta de modificación al Reglamento de Zonificación vigente, 2008). 
A este panorama se agregan características atractivas para los procesos metropolitanos: disponibilidad de suelos accesibles a precios menores que en la ciudad central y a la vez consumo del suelo productivo.

En atención a lo anterior, el texto siguiente describirá esa dinámica en función del reconocimiento de la escala urbana, pero también de la metropolitana, estrechamente vinculada con la lógica de la circulación y de los intercambios.

El impacto de las transformaciones descritas en las localidades aglomeradas se puede simplificar en dos tendencias: por un lado, un crecimiento tradicional en ellas, en continuidad con el núcleo existente y sin mayores alteraciones del tejido consolidado; por otro, una ocupación a lo largo de las vías de comunicación, que va entretejiendo usos diversos y cambiando radicalmente estos perfiles rururbanos ${ }^{6}$.

En respuesta a esta segunda tendencia de extensión, la cual está ligada principalmente al desborde de usos por parte de la ciudad central (Santa $\mathrm{Fe}$ ), suponemos que es clarificador presentar un desglose de este proceso, explicitado para cada uno de los ejes de crecimiento.

\section{Eje de expansión metropolitana noreste: Ruta Provincial $N^{0}$ I}

Este eje - también llamado Corredor de la Costa, por tratarse de un albardón casi insular - representa la expansión residencial más significativa del Gran Santa Fe a través de la Ruta Provincial Nº 1 (RP No 1), la cual vertebra las localidades de la costa y comunica con el norte de la provincia.

En este sector se reconoce una expansión acelerada que comenzó en la localidad de San José del Rincón hasta casi colmatarla. En los últimos años, tal como se observa en la figura 3 , el crecimiento relativo se ha enfatizado en la localidad de Arroyo Leyes y ha alcanzado un segundo avance de extensión puramente residencial, difuso, de baja densidad y con condiciones de riesgo hídrico.

6 Lo rururbano tiene que ver con una categoría del análisis de los procesos urbanos que intenta asir esa mixtura típica de las zonas donde se ha iniciado un proceso de ocupación de carácter urbano, pero que aún conservan una impronta rural con una oferta limitada de servicios y equipamientos. 
Para alentar esta tendencia, se ha desarrollado recientemente la obra de conversión en autovía de esta ruta en un primer tramo (hasta el kilómetro 6,6$)^{7}$.

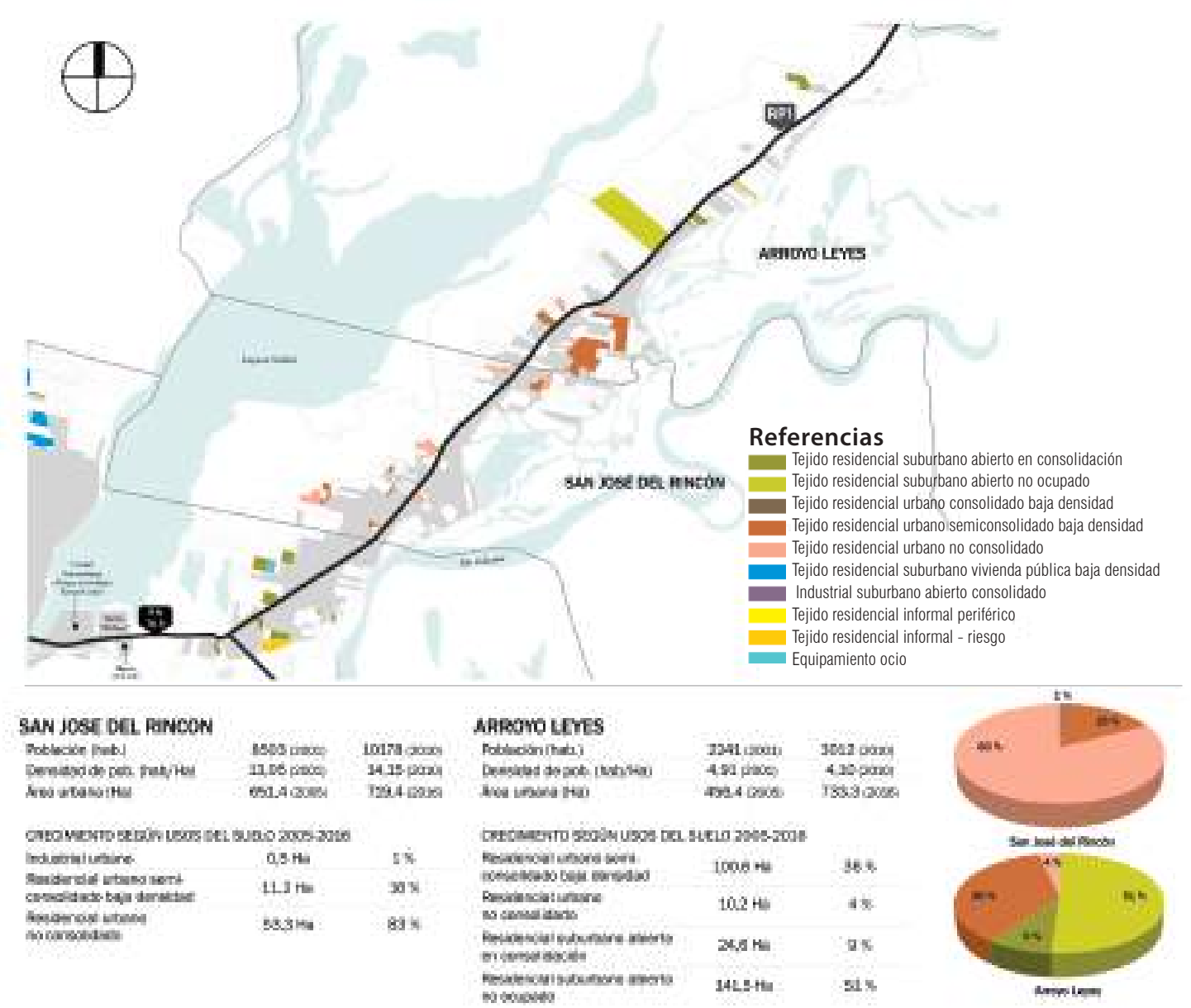

Figura 3

Esquema que muestra la extensión de usos del suelo en Rincón y en Arroyo Leyes (2005-2016)

Fuente: Google Earth. Indec

Elaboración propia

7 Además de otros fines, como la consolidación de un corredor comercial y servicios de escala local/cotidiana y regional, aún resta un periodo de evaluación de las mejoras en el acceso a las localidades aglomeradas. 
Ejes de expansión metropolitana norte: Ruta Nacional № II y Ruta Provincial № 2

La expansión norte se manifiesta como un desgranamiento por el cual la ciudad central, sigilosamente y durante años fue desbordando su límite jurisdiccional, principalmente en direcciones tensionadas por la presencia de las vías de conexión territorial Ruta Nacional $N^{\circ} 11$ (Recreo) y Ruta Nacional Nº 2 (Monte Vera).

El desborde de Santa Fe sobre el corredor Ruta Nacional No 11 (RN N 11) se fue consolidando de manera lineal, siguiendo la traza de la ruta, y generó lo que comúnmente se llama Recreo Sur, una conurbación entre Santa Fe y Recreo. La existencia de la Autopista Santa Fe - Rosario refuerza esta situación hacia el sur; y la incorporación del tramo III de la Circunvalación Oeste hacia Recreo (que también constituye un terraplén de defensa del río Salado) agiliza la circulación hacia el norte y permite desviar el tránsito pesado de las áreas urbanas de ambas localidades ${ }^{8}$.

En lo que respecta a los equipamientos, esta zona noroeste va configurando un perfil industrial-logístico con el Mercado de Productores y la zona industrial Los Polígonos en Santa Fe; además, se proyecta la construcción de un área logística y un parque industrial en Recreo.

Si bien se advierten algunas situaciones de completamiento de la planta urbana de Recreo, se puede ver que la mayor extensión ocurre de forma suburbana y residencial hacia la Circunvalación Oeste, lo cual ha posibilitado un nuevo eje de penetración desde Santa Fe, sumado al ya existente (RN No 11).

Como se puede observar en la figura 4, la localidad de Recreo incrementó su planta urbana en aproximadamente un $30 \%$ y pasó de 613,6 Ha en el 2005 a 806,4 Ha en el 2016. Casi un $80 \%$ de este crecimiento se registró al oeste de la planta urbana tradicional, a partir de la incorporación de un extenso loteo propiciado por los créditos del Programa de Crédito Argentino del

8 En la actualidad, la RN N ${ }^{0} 11$ cuenta con un proyecto de autopista hacia la ciudad de San Justo, paralela a esta ruta y como prolongación de la Circunvalación Oeste. Esta obra produciría grandes beneficios en el tránsito y acentuaría la condición estructural que actualmente tiene este eje. 
Bicentenario para laVivienda Única Familiar (Procrear) ${ }^{9}$. Estos contribuyeron al loteamiento y ocupación de loteos de estas características: periféricos, de baja densidad, con pocos o nulos servicios, y en muchos casos desconectados.

Paralela a la RN No 11 se encuentra, hacia el este, la RP $N^{\circ} 2$, en cuyas márgenes se desarrolla el cordón frutihortícola Santa Fe-Monte Vera, que hoy se encuentra muy reducido debido a la expansión urbana del norte santafesino. El distrito Monte Vera, junto con la localidad y los parajes que lo integran, está sufriendo las consecuencias —en términos de consumo de suelo productivo - de la expansión urbana difusa y de baja densidad, a través de la proliferación de urbanizaciones residenciales que son producto de grandes operaciones privadas.

De las nuevas urbanizaciones, la mayor extensión se reconoce en el gran loteo Las Moras, al oeste de la planta urbana; pero también se advierte que los pequeños parajes presentan dinámicas propias de crecimiento.

Como se puede observar en la figura 4, esta localidad extendió su planta urbana en aproximadamente un $50 \%$ y pasó de 230,9 Ha en el 2005 a 363,1 Ha en el 2016. Si bien el loteo Las Moras representa un $25 \%$ de esta extensión, el crecimiento del tejido suburbano de los parajes es superior, sobre todo en Ángel Gallardo, que se encuentra más cercano a la ciudad de Santa Fe y absorbe parte de la demanda residencial.

En cuanto a densidades, ambas localidades registraron una disminución, lo que responde al crecimiento disperso y en baja densidad que experimentaron. Así, en Recreo la densidad pasó de 20,85 hab./Ha a 17,61 hab./Ha, y en Monte Vera pasó de 30,61 hab./Ha a 22,81 hab./Ha.

9 En Procrear se previó el otorgamiento de créditos para la construcción de hasta 400000 viviendas populares entre el 2012 y el 2016. Se trata de un proyecto realizado y gestionado por la Presidencia de la Nación, en acción conjunta con la Anses (Administración Nacional de la Seguridad Social), el Ministerio de Economía y Finanzas Públicas, y el Banco Hipotecario. El programa continúa, pero se han reformulado algunos aspectos. 


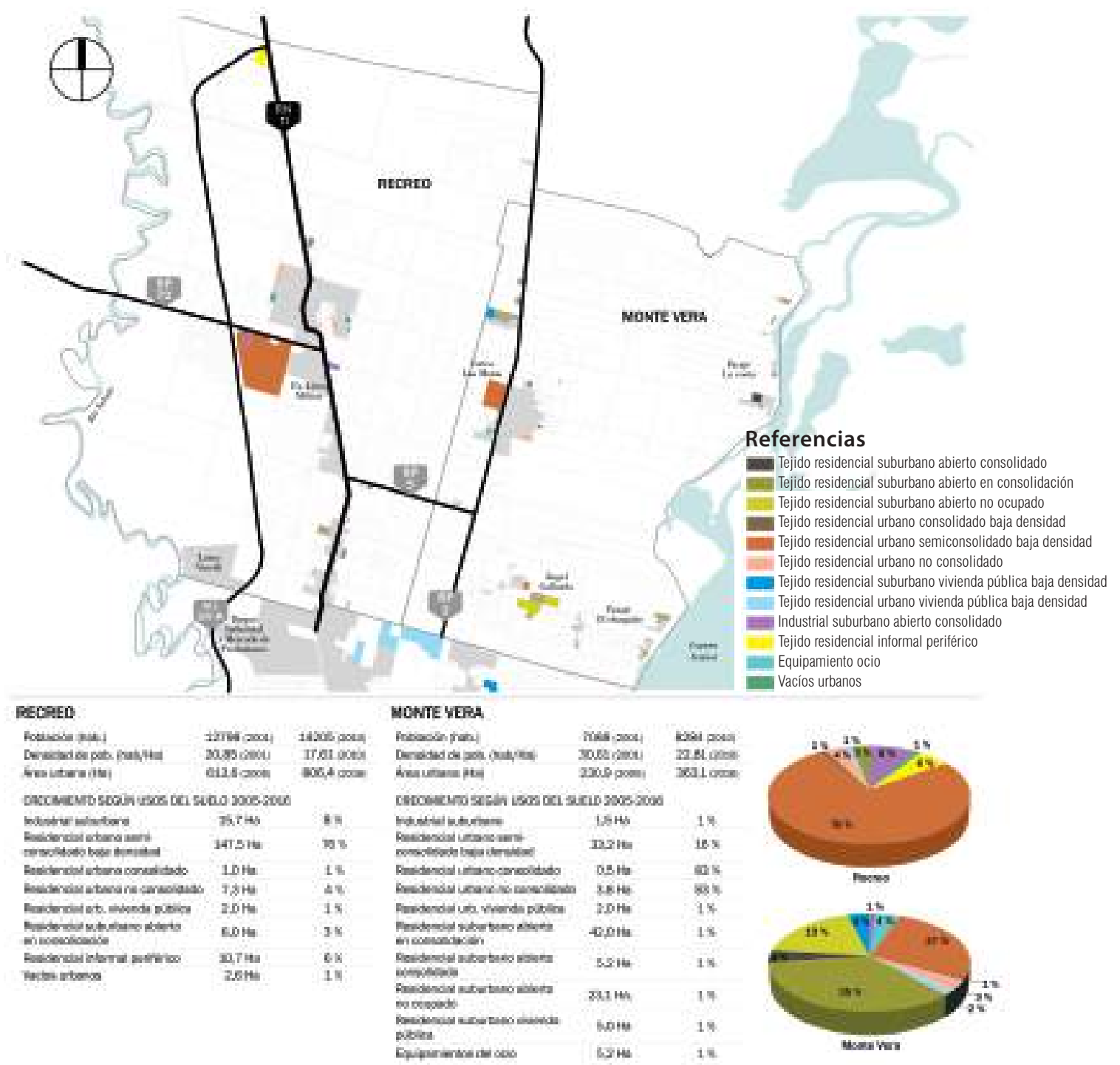

Figura 4

Esquema que muestra la extensión de usos del suelo en Recreo y Monte Vera (2005-2016)

Fuente: Google Earth. Indec

Elaboración propia 


\section{Ejes de expansión sudoeste: Ruta Nacional No 19 y Ruta Nacional No II (autopista APOI)}

Al oeste, la Ruta Nacional No 19 (RN No 19) —recientemente transformada en autovía - constituye un corredor nacional que conecta las capitales provinciales de Santa Fe y Córdoba; además, su alcance es internacional (Chile y Brasil), ya que forma parte del Corredor Bioceánico Central.

Respecto del entorno inmediato del Gran Santa Fe, este corredor atraviesa la ciudad de Santo Tomé y posee una significativa cantidad de asentamientos industriales. El Plan Director de Santo Tomé (Ordenanza 818) en 1979 habilitó un distrito destinado a área industrial, pero demandas muy posteriores generaron procesos de consolidación. Estos comenzaron aproximadamente en el 2000 y continúan en la actualidad, como se observa en la figura 5 . Tales dinámicas territoriales explican localizaciones, por desborde de equipamientos, infraestructuras, usos y demandas específicas, que la ciudad central por diferentes motivos no ha absorbido.

En terrenos aledaños a la autopista Santa Fe-Rosario, la extensión que avanza sobre el ejido de la ciudad de Santo Tomé — en la margen opuesta del río Salado - tiene que ver con la tendencia hacia la periferia residencial suburbana, vinculada a la lógica de los enclaves: clubes de campo o complejos recreativos residenciales, y barrios cerrados ${ }^{10}$, todos desarrollados en grandes parcelas. Como puede verse en la figura 5, este uso lidera la expansión suburbana y urbana santotomesina y representa el $71 \%(161,7 \mathrm{Ha})$ del área de extensión.

Hacia el sur se encuentra la ciudad de Santo Tomé, la más grande en población y extensión de las aglomeradas del sistema Gran Santa Fe, y la que registra mayor densidad demográfica $(33,09$ hab./Ha) después de Santa Fe. Si bien esta localidad surgió como ciudad dormitorio de Santa Fe, en la actualidad presenta dinámicas propias y su incremento poblacional no solo responde al desborde santafesino, sino también al crecimiento propio.

10 Estos fueron habilitados por legislación municipal sancionada en 1987 (Ordenanza 1337) y 2003 (Ordenanza 2390), que permitió uno y otro tipo de urbanización, respectivamente. 


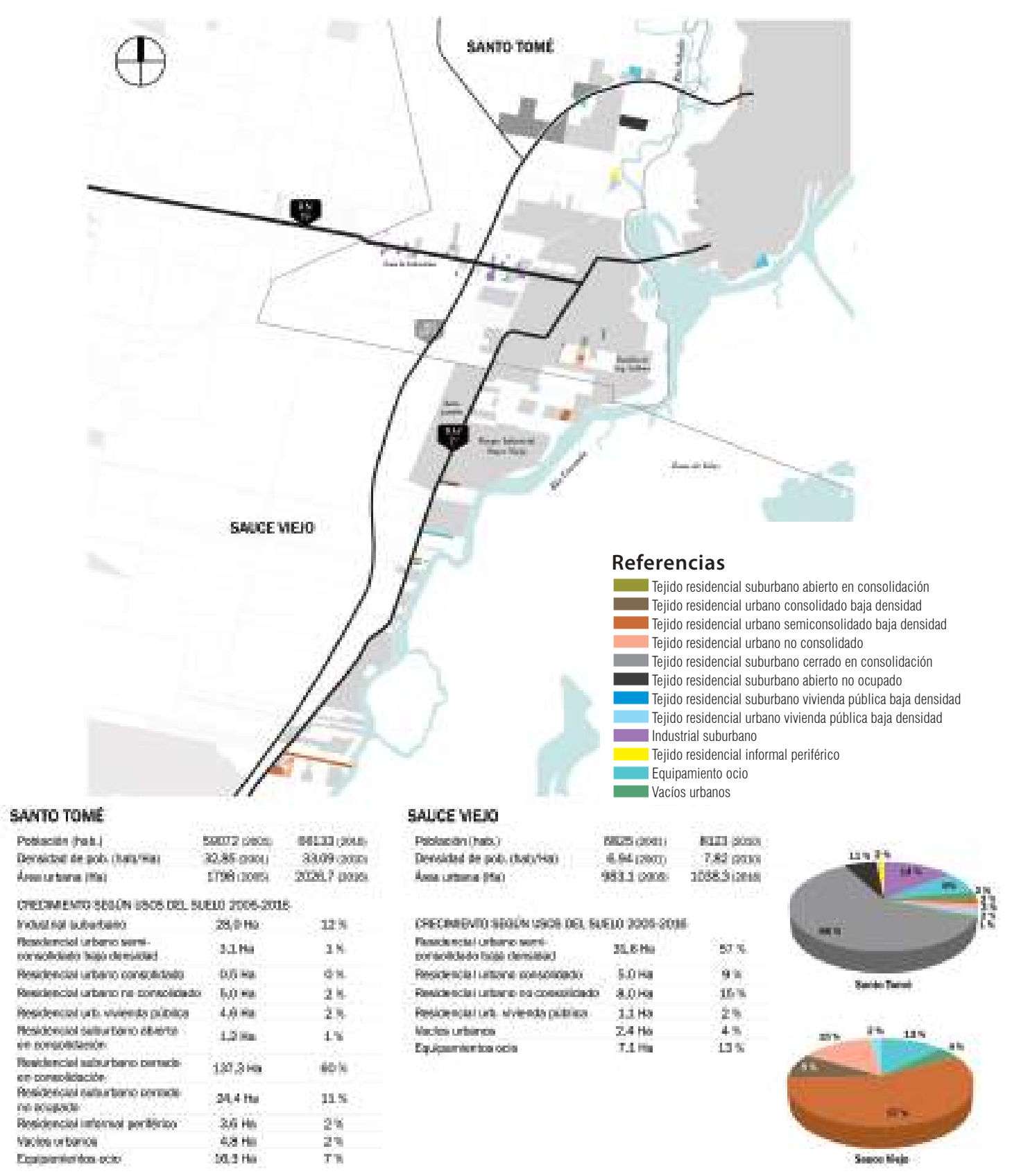

Figura 5

Esquema que muestra la extensión de usos del suelo en Santo Tomé y Sauce Viejo (2005-2016)

Fuente: Google Earth. Indec

Elaboración propia 
Dentro de este corredor se considera también la autopista Santa Fe-Rosario (AP01) y la continuidad de la RN N 11 hacia el sur, atravesando la localidad de Sauce Viejo. Destaca la presencia de equipamientos especiales (antiguos y fundacionales), de escala metropolitana, como el aeropuerto provincial (1955) y el parque industrial de $172 \mathrm{Ha}$ (1973), además del proyecto en cartera de un parque comercial mayorista; todos ubicados en la jurisdicción de Sauce Viejo. En su entorno se ha ido generando una mixtura de usos, entre los que se incluyen áreas vacantes, comercios de diverso tipo, talleres, industrias, producción agrícola y frutihortícola, lugares de recreación, y áreas residenciales con características de barrio jardín que - no obstante tal carácter "unificador"- muestran sectores diferenciados en función de los grupos socioeconómicos que los habitan. Hay que destacar que los usos residenciales suburbanos completan amplios sectores de esta expansión. En un primer momento esas viviendas habían sido concebidas como predominantemente finisemanales o transitorias; sin embargo, en las últimas décadas han tenido mayor demanda de semipermanencia o permanencia, sobre todo con la proliferación de los créditos Procrear. Sin embargo, como se vio en la figura 5 , no se manifiestan significativas incorporaciones de suelo residencial como en las localidades del noreste, puesto que se identifica una incorporación de apenas 55,2 Ha entre el 2005 y el 2016. Dicha cifra representa una extensión del 5,6\% de la planta urbana, debido a que el suelo ya se encontraba subdividido pero no ocupado. A su vez, esta localidad registra una de las densidades más bajas de todo el aglomerado, con un valor de 7,82 hab./Ha.

\section{CONCLUSIONES: CONSOLIDACIÓN VERSUS FRAGMENTACIÓN}

Se ha observado un proceso de gran contraste en el territorio que tiene que ver, por un lado, con la consolidación y densificación de la ciudad central Santa Fe; y por otro, con la situación de crecimiento extensivo y disperso en las localidades aglomeradas y las zonas periféricas de la propia ciudad.

De esta forma, el territorio de la metrópoli se disgrega en un conglomerado de unidades yuxtapuestas, pero separadas, producto espontáneo de un proceso natural de autosubdivisión de unidades homogéneas, diferenciadas del conjunto. 
En tal sentido se puede hacer referencia al concepto de ciudad difusa o, mejor aún, a un tipo de ciudad difusa que surge como espacialización de las diversidades y la planificación ausente o parcial que, en buena medida, caracterizan lo urbano en Latinoamérica. Este proceso de metropolización se distingue por la extensión de los movimientos pendulares diarios alrededor de las grandes ciudades, lo que lleva al crecimiento de áreas periurbanas, la urbanización policéntrica, la ocupación difusa sobre el suelo rural, la polarización social, la segregación residencial y la fragmentación de la estructura urbana. Todos ellos son rasgos destacados de una nueva geografia.

En ese sentido, Rausch (2011) describe la situación periférica santafesina como "un estado de urbanización del territorio rural que no puede considerarse en términos de ciudad y que establece un nuevo sistema de jerarquías, tanto en la tradicional relación ciudad-campo, como en su conformación interna (al territorio)". La demanda residencial incentivada, además, por políticas públicas específicas ha contribuido a la dispersión, no en la categoría de campos urbanizados, sino como urbanizaciones aisladas o contiguas, y fragmentos formalmente completos, cuya consolidación va dejando entre ellos grandes espacios de ruralidad, a la vez que expande los límites de lo considerado urbano.

Entre los factores determinantes de esta modificación destaca el crecimiento demográfico y físico de la ciudad de Santa Fe. Ha cubierto casi la totalidad del suelo de su jurisdicción con usos urbanos y ahora demanda de las localidades vecinas el soporte territorial para seguir potenciando su crecimiento. Así es como el crecimiento se orienta predominantemente a una tendencia expansiva e instala un proceso de conurbación que evidencia fuertes vinculaciones entre la ciudad central y los distritos aledaños, como se da claramente con Recreo y Monte Vera al norte. Estas localidades han acelerado la división de sus suelos y han mutado los usos productivos predominantes a nuevos usos residenciales (Soijet, Mantovani y Peralta, 2016).

A partir de investigaciones desarrolladas en el marco del Observatorio Urbanístico AMSF-P, se ha llegado a la conclusión de que el paisaje periférico resultante es un patchwork de viviendas unifamiliares de gestión pública y privada; equipamientos recreativos, deportivos, industriales; grandes instalaciones de servicios de escala metropolitana y regional; 
parcelas de producción frutihortícola; áreas de servicios, y grandes trazados infraestructurales.

En síntesis, este proceso tiene un comienzo de equilibrio urbano-rural con la ciudad inserta en un área agrícola y continúa con la difusión de asentamientos urbanos rodeados de áreas rurales. Sigue un momento posterior de difusión de la urbanización con consumo de esas áreas productivas en anillos sucesivos alrededor de las ciudades más importantes, lo que se define hoy como una gran mancha continua que presenta puntos de intensidad diversa de urbanización, con pocas áreas de campo incorporadas y cierta tendencia a la aglomeración.

\section{PROPUESTA: REDES PARA UN EQUILIBRIO DEL TERRITORIO}

No quedan dudas de que buena parte de los problemas descritos están vinculados, ya sea porque se originan o porque se agravan, a los procesos de dilatación de la ciudad. Carlos Llop (2008, acápite 3, párr. 3) lo sintetiza así:

Extensión de las formas de ciudad sobre el territorio con la disipación de funciones que eso conlleva, dispersión residencial sobre ámbitos territoriales cada vez más alejados de los centros, polarización de funciones centrales en los nudos de accesibilidad metropolitana, grandes transformaciones internas de la ciudad consolidada, aumento de los perímetros periurbanos, congestión de las infraestructura y problemas de uso de determinados suelos.

Estamos frente a una serie de fenómenos que se están produciendo en muchas ciudades, y no solo de la Argentina. Es el crecimiento extralimitado, desbordado de la ciudad, sin planificación, y que se extiende sobre zonas que tienen otros usos, como sistemas de producción de alimentos, o que deberían ser reservas, porque favorecen la sustentabilidad (pedemontes, selvas en galería, yungas) o aprovisionan a los sistemas infraestructurales.

Una posible agenda que lleve a abordar tan compleja situación parece dilucidarse a través de vínculos de nodos interdisciplinares e interestamentales en red.Estas herramientas, con nombres diferentes (observatorios, monitoreos, laboratorios, seguimientos) pueden ser locales, a partir de alianzas con actores territoriales que identifican integralmente los problemas y buscan soluciones; y también, en un ámbito más general, pueden ser puestas en agendas 
gubernamentales e institucionales con el objeto de poner en valor ciertas áreas como una forma de proteger paisajes, cadenas de valores, soberanía alimentaria, etcétera.

Para conformar estas redes, se debería apuntar a una agenda temática que, a partir de la experiencia que presentamos, incluya una serie de acciones que vienen siendo objeto de indagación:

- Dotar de transparencia al mercado de suelos al permitir reconocer sus actores y su dinámica. Hoy, esta información está protegida por una serie de confidencialidades que no disponen otras informaciones.

- Considerar las actividades extractivas, en las que el recurso suelo es un material directo para la construcción (ladrilleras, tosqueras, etcétera).

- Definir políticas conjuntas desde lo urbano-residencial y lo ruralproductivo. Esto implica que cualquier normativa urbanística deberá considerar políticas sectoriales y territoriales hortícolas (locales y macrorregionales) para definir cinturones verdes, parques agrícolas, etcétera.

- Definir políticas conjuntas y coordinadas en materia de movilidad territorial y urbana en articulación con las redes de servicios, en las que se determinen actuaciones con algún grado de integralidad y que apunten al funcionamiento coordinado de personas, bienes y prestaciones.

- Dotar de capacidades y fortalecer las gestiones locales para que puedan actuar frente a otros actores, como los desarrolladores. Estos fomentan la instalación de urbanizaciones cerradas y están conformando un mercado muy potente y dificil de detener con la idea de recorte de la periferia (enclave) para los sectores más acomodados de la sociedad.

En relación con estas tres últimas cuestiones, resulta importante mencionar que la Provincia de Santa Fe ha promulgado en el 2016 la Ley Provincial 13.532, de Creación de Áreas Metropolitanas, y se encuentra en proceso de elaboración de lineamientos para el caso de Santa Fe en el ámbito metropolitano. Esos lineamientos establecen como temas prioritarios la definición de estrategias para la movilidad, los usos del suelo y el riesgo. 
Frente a la velocidad de las transformaciones recientes, es necesario y oportuno profundizar en cada una de estas propuestas, con una fuerte base analítica-propositiva desde el campo disciplinar, pero con miras a su enriquecimiento a partir de la interdisciplina, así como a su instrumentación en un esquema operativo interinstitucional. Todo ello deja abierto un campo de trabajo que será objeto de nuestra atención particularizada en la continuidad de las indagaciones.

\section{REFERENCIAS}

Castreghini de-Freitas-Firkowski, O., y Casares, M. (2016). Curitiba-San Miguel de Tucumán: posibilidades e límites de pesquisa comparada. En O. L. Castreghini de Freitas-Firkowski, M. D. Casares Madianita Nunes da Silva, N. P. Czytajlo y D.Volochko Freitas-Firkowski (coords.), Estudios urbanos comparados (pp. 123-150). San Miguel de Tucumán: Universidad Nacional de Tucumán.

Fein, M. (2014) El área metropolitana de Rosario: estructura institucional y caracterización territorial. Rosario: ECOM. En Programa de Desarrollo de Áreas Metropolitanas del Interior. Rosario: BID.

Gobierno de Santa Fe. (2016). Ley Provincial 13.532 de creación de Áreas Metropolitanas de Santa Fe. Recuperado de https://www.santafe.gov. ar/index.php/web/content/download/228816/1198134/file/Ley\%20 de\%20Areas\%20Metropolitanas.pdf

Llop, C. (2008). Paisajes metropolitanos: policentrismo, dilataciones, multiperiferias i microperiferias. En Revista Papers. Institut d'Estudis Regionals i Metropolitans de Barcelona, El repte del paisatge en àmbits metropolitans, (47), 8-13.

Martínez, I. (2016). Verticalización del núcleo urbano central de Santa Fe: sus representaciones sociales entre 1966 y 2010. Revista Arquisur, 6(9), 90-105.

Ministerio de Planificación Federal, Inversión Pública y Servicios. (2011). Plan Estratégico Territorial Avance II: Argentina Urbana. Buenos Aires: Autor. 
Rausch, G. (2011). Una historia periférica. El proceso de urbanización en el valle aluvial santafesino. Revista Polis, (10).

Reese, E. (2009). Entre el plan y el mercado. La formación de una nueva agenda de las ciudades en la Argentina (ficha interna del curso de grado Urbanismo 2 de FAUD-UNMDP 2013).

Soijet, M., Mantovani, G., y Peralta-Flores, C. (2016). Interfase de transición urbano-territorial: el reconocimiento de una nueva categoría estructural y su operatividad. El caso del Área Metropolitana Santa Fe-Paraná. En Universitat Politècnica de Catalunya y Universidad del Valle de Itajaí (Ed.), VIII Seminario Internacional de Investigação Em Urbanismo, Cidade, Território e Paisagem: Pesquisa e Projeto. Universidad de Barcelona.

Universidad Nacional del Litoral. (2014). Proyecto de Extensión de Interés Social. "Afrontando los problemas de la ciudad con herramientas innovadoras". (Archivo de video). Santa Fe:Autor. 